\title{
Comparison of the 16S-23S rRNA Intergenic Spacer Regions between Fusobacterium varium and "Fusobacterium pseudonecrophorum" Strains
}

\author{
Jinghua JIN ${ }^{1)}$, Wonganun NARONGWANICHGARN ${ }^{1)}$, Delong XU' ${ }^{1)}$, Yoshitaka GOTO ${ }^{1)}$, Takeshi HAGA ${ }^{1)}$ and \\ Toshiharu SHINJO')*

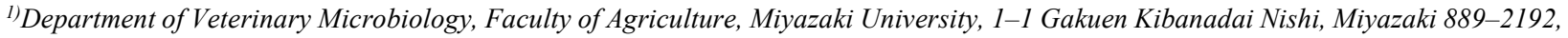 \\ Japan
}

(Received 21 June 2001/Accepted 12 December 2001)

ABSTRACT. The 16S-23S rRNA intergenic spacer regions (ISRs) of five strains of "Fusobacterium pseudonecrophorum" which had been proposed as a new species, were compared with those of $F$. varium ATCC $8501^{\mathrm{T}}$. All the strains of " $F$. pseudonecrophorum" exhibited of sequence similarities of $97.7 \%$ to $100 \%$ to the strain of $F$. varium in their $16 \mathrm{~S}-23 \mathrm{~S}$ rRNA ISR sequences. This indicates that the strains of " $F$. pseudonecrophorum" and the type strain of $F$. varium are identical at the species level.

KEY WORDS: Fusobacterium varium, ISR, 16S-23S rRNA.

J. Vet. Med. Sci. 64(3): 285-287, 2002

We proposed "Fusobacterium pseudonecrophorum" as a new species for the biovar $\mathrm{C}$ of $F$. necrophorum based on the DNA-DNA homology analysis [12]. Recently, Bailey and Love [3] reported that " $F$. pseudonecrophorum" and $F$. varium were specifically related to each other from the results of DNA-DNA hybridization analysis. In their report, they proposed that "F. pseudonecrophorum" was a synonym of $F$. varium. However, the isolation sources and pathogenicity of the two species differed from each other [11]. The former was isolated from the lesions of various human organs and was experimentally pathogenic to the guinea pig and rabbit, while the latter was less pathogenic to these animals.

The 16S rDNA sequence is now well known and is applied in both phylogeny and the identification of prokaryotes. It is used for analysis at the genus level, and its intrachromosomal differences are small and can be dismissed in phylogenetic analysis. In addition, DNA analysis of the $16 \mathrm{~S}-23 \mathrm{~S}$ rRNA intergenic spacer region (ISR) was shown to be very useful in typing and identification of bacteria [5]. The ISR analysis is a more useful tool for closely related organisms such as Listeria and Staphylococcus than the 16S rRNA analysis because of its precise differentiation ability [5-7]. To date, limited data are available concerning the sequence analysis for fusobacterial species. Therefore, we evaluated the propriety of the new classification using the nucleotide sequences of the 16S-23S rDNA ISR among $F$. varium ATCC $8501^{\mathrm{T}}$ and five " $F$. pseudonecrophorum" strains.

Six strains including the type strain ATCC $8501^{\mathrm{T}}$ of $F$. varium and strains JCM3721, JCM3722, JCM3723, Fn525 and Fn526 of " $F$. pseudonecrophorum" were used in this study. All of these strains were inoculated to GAM agar (Nissui Seiyaku Co., Tokyo) supplemented with 5\% horse

\footnotetext{
* Correspondence to: Shinjo, T., Department of Veterinary Microbiology, Faculty of Agriculture, Miyazaki University, 1-1 Gakuen Kibanadai Nishi, Miyazaki 889-2192, Japan.
}

blood and incubated anaerobically by the steel wool method at $37^{\circ} \mathrm{C}$ for $72 \mathrm{hr}$ [1]. The developed colonies were subcultured in BPPY medium [10] at $37^{\circ} \mathrm{C}$ for $24 \mathrm{hr}$, and the cultures were used for this study. Bacterial DNA was isolated and purified by phenol/chloroform extraction and ethanol precipitation [2].

PCR amplification of the 16S-23S rRNA ISR was performed with the following primers: the forward primer Ec16S, 1390p, 5'-TTG TAC ACA CCG CCC GTC-3' [4]; and the reverse primer Ec23S, 441p, 5'-TAC TGG TTC ATA ATC GGT CA-3' [9]; having been described elsewhere. The PCR products were purified with a QIA quick PCR Purification Kit (QIAGEN) according to the manufacturer's instructions. The nucleotide sequences of the purified PCR products were determined by the direct sequencing method and aligned by using the CLUSTAL W program [13].

Two bands of PCR products (long and short) were observed for all the strains examined. The amplified products approximated to $700 \mathrm{bp}$ and $800 \mathrm{bp}$ in length. The short bands (ca. $700 \mathrm{bp}$ ) showed higher intensities than the long ones (ca. $800 \mathrm{bp}$ ). Only the short bands of the two PCR product sequences were used for the sequence analysis. According to the rDNA sequence of $F$. varium, available in the DDBJ/EMBLE/GENBANK (accession number AF342853), the 5' and 3' termini of the 16S-23S rRNA ISRs were specifically located as shown in Fig. 1. The lengths of the ISR from the shorter PCR products were subsequently found to be $171 \mathrm{bp}$ for $F$. varium ATCC $8501^{\mathrm{T}}$ and $173 \mathrm{bp}$ for the five strains of " $F$. pseudonecrophorum" $F$. varium and "F. pseudonecrophorum" strains exhibited similarities of $97.7 \%$ to $100 \%$, based on the alignment shown in Fig. 1. For estimating the copy number of the $16 \mathrm{~S}$ rDNA in all strains, the genomic DNAs were digested with the restriction enzyme HindIII and then applied to Southern hybridization with PCR-amplified $16 \mathrm{~S}$ rDNA of $F$. varium ATCC $8501^{\mathrm{T}}$ as a probe. All strains were shown to have two 
3' end of $16 \mathrm{~S}$ rRNA gene

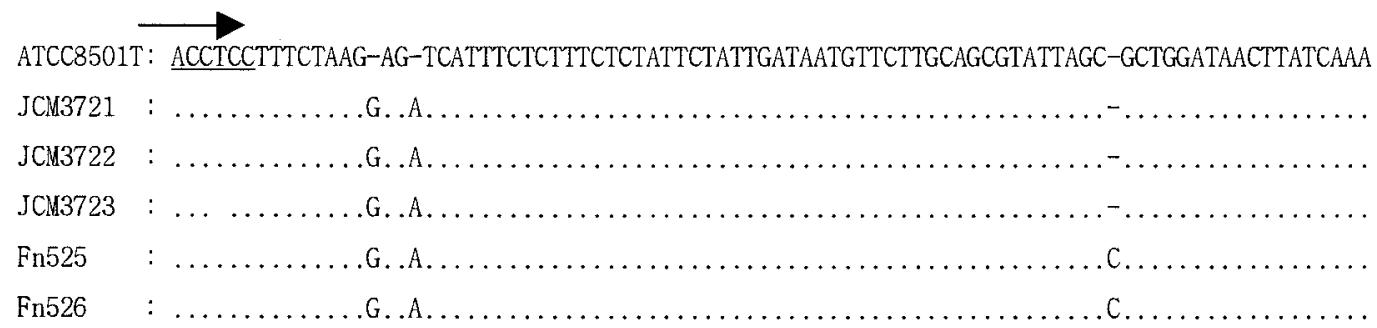

ATCC8501T: TGGACATTGGAAACTATATAGTAGATAATACAATTTTAACTCTTGTTTATATAAACAGAGTTAGCTGTCAATCAAATTAAACAAAA

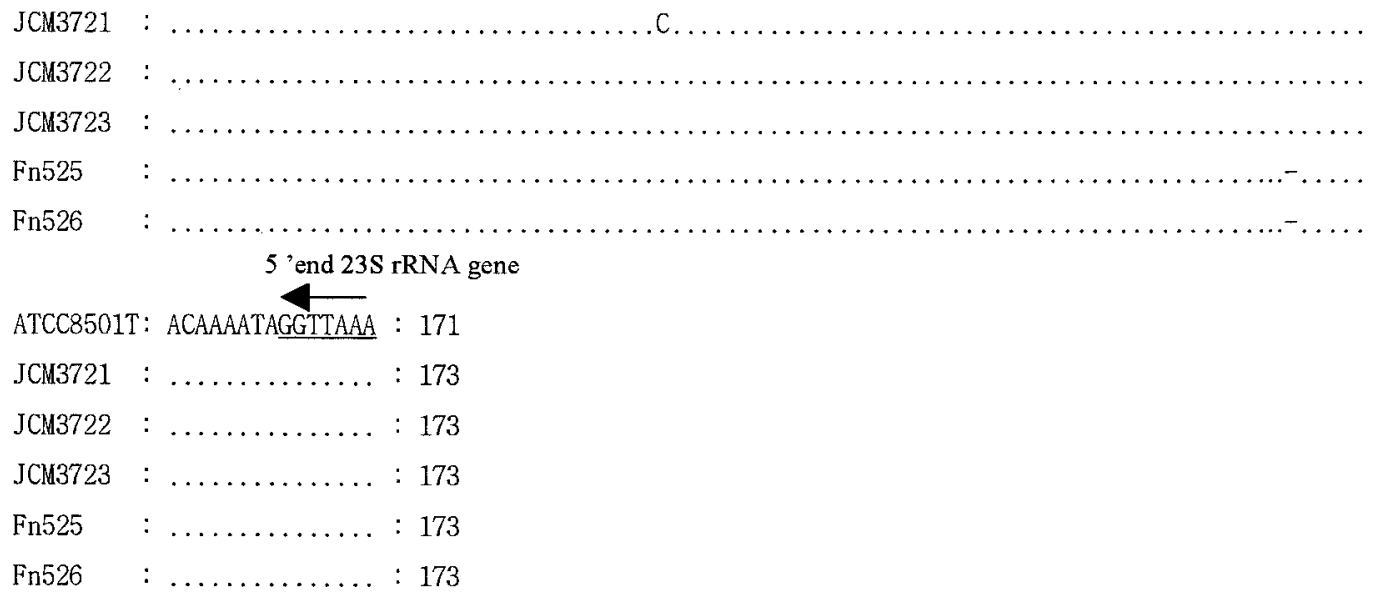

Fig. 1. Alignment of 16S-23S ISR sequences of $F$. varium ATCC $8501^{\mathrm{T}}$, and " $F$. pseudonecrophorum" strains, JCM3721, JCM3722, JCM3723, Fn525, and Fn526. The length of the ISR is noted at the end of the sequences. The complete ISR sequence between the end of the 16S rRNA gene and the beginning of the 23S rRNA gene is shown. Nucleotides, identical to $F$. varium ATCC $8501^{\mathrm{T}}$ are marked by dots. Hyphens represent alignment gaps.

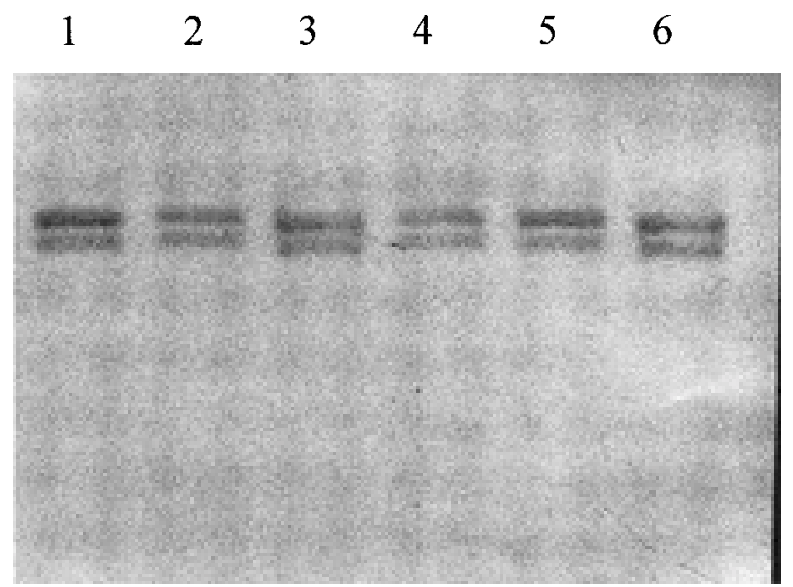

Fig. 2. Southern hybridization of $F$. varium and " $F$. pseudonecrophorm" with the PCR-amplified 16S rDNA of $F$. varium ATCC $8501^{\mathrm{T}}$ as a probe. The genomic DNA was digested with HindIII. 1. F. varium ATCC $8501^{\mathrm{T}}$, "F. pseudonecrophorum" strains JCM3722 (2), JCM3723 (3), JCM3721 (4), Fn525 (5), and Fn526 (6). copies of rDNA alleles on their genomes (Fig. 2). However, there was no tRNA-like structure within the ISR sequences as shown in Fig. 1 when the tRNAscan-SE program [8] was used.

In conclusion, no significant difference at the species level was observed between $F$. varium and " $F$. pseudonecrophorum" on the basis of sequence homologies in the $16 \mathrm{~S}-23 \mathrm{~S}$ rRNA ISR.

\section{REFERENCES}

1. Amoako, K. K., Goto, Y. and Shinjo, T. 1993. J. Clin. Microbiol. 31: 2244-2247.

2. Ausubel, F. M., Brent, R., Kingston, R. E., Moore, D. D., Seidman, J. G., Smith, J. A. and Struhl, K. 1987. pp. 2.3.22.4.1. In: Current Protocols in Molecular Biology, Wiley, New York.

3. Bailey, G. D. and Love, D. N. 1993. Int. J. Syst. Bacteriol. 43: 819-821.

4. Frothingham, R. and Wilson, K. H. 1993. J. Bacteriol. 175: 2818-2825.

5. Gürtler, V. and Stanisich, V. A. 1996. Microbiology 142: 3-16.

6. Jenson, M. A., Webster, J. A. and Straus, N. 1993. Appl. Envi- 
ron. Microbiol. 59: 945-952.

7. Leblond-Bourget, N., Philippe, H., Mangin, I. and Decaris, B. 1996. Int. J. Syst. Bacteriol. 46: 102-111.

8. Lowe, T. and Eddy, S. R. 1997. Nucl. Acids Res. 25: 955-964.

9. Meijer, A., Kwakkel, G. J., Vries, A., Schouls, L. M. and Ossewaarde, J. M. 1997. J. Clin. Microbiol. 35: 1179-1183.

10. Miyazato, S., Shinjo, T., Yago, H. and Nakamura, N. 1978.
Jpn. J. Vet. Sci. 40: 619-621.

11. Prévot, A.-R. 1957. Manuel de Classification et de Détermination des Bactéries Anaérobies, Masson et $\mathrm{C}^{\mathrm{ie}}$., Paris.

12. Shinjo, T., Hiraiwa, K. and Miyazato, S. 1990. Int. J. Syst. Bacteriol. 40: 71-73.

13. Thompson, J.D., Higgins, D. G. and Gibson, T. J. 1994. Nucl. Acids Res. 22: 4673-4680. 